\title{
Three-Dimensional Reconstruction and Quantification of PGP9.5-Immunoreactive, Sensory Intra-Epidermal Nerve Fibers
}

\author{
Michael Anderson ${ }^{1}$ and Kenneth Miller ${ }^{1}$
}

1. Oklahoma State University Center for Health Sciences, Anatomy and Cell Biology, Tulsa, OK.

Intra-epidermal nerve fibers (IENFs) are the peripheral component of primary sensory neurons and are the anatomical attributes for an organism's ability to sense the environment, i.e., tactile, temperature stimuli. These small, unmyelinated, IENFs also transmit protective noxious information critically important to the survival of an organism. Decrease of IENFs within peripheral tissue may indicate potential disease. Several life-threatening peripheral neuropathies, such as autoimmune diseases and diabetes, can be assessed with a skin biopsy. Modern skin biopsy techniques involve removing a small portion of skin to evaluate IENF density and branching. It has been reported that IENF swelling, decrease in IENF density, increased branching, and morphological fragmentation of intra-epidermal nerves may indicate potential disease [1].

IENFs branch throughout the epidermis in three-dimensions (3D). Traditional quantitative evaluation of intra-epidermal nerves involve: tissue collection, fixation, sectioning, immunohistochemistry (IHC), and tracing/quantification of two-dimensional (2D) images. This technique has been the industry standard for quantification of intra-epidermal nerves in biomedical research and the limitations have largely been accepted.

A novel, efficient, and cost-effective technique for 3D IENF quantification would provide a powerful tool in the study of peripheral nerves. Quantifying 3D objects with 3D measurement provides greater resolution of data per individual nerve fiber. The challenges of $3 \mathrm{D}$ IENF imaging and quantification include: epidermal isolation, rendering tissue optically transparent for imaging of z-depth, penetration of antibody with fluorescent labels, proper imaging at greater depths $(60-180+\mu \mathrm{m})$, and software segmentation and quantification.

Intra-epidermal nerve isolation was achieved by the use of thermolysin from Geobacillus stearothermophilus at $4{ }^{\circ} \mathrm{C}$ for approximately 2 hours [2]. The low temperature of enzyme activity for thermolysin improves protein integrity before it can be preserved in a tissue fixative. Optical transparency of isolated epidermis is achieved through a modified tissue-clearing (TC) technique [3]. This involves plasticizing the isolated epidermis in acrylamide at a concentration that allows for increased tissue integrity and molecule penetration for IHC fluorescent labeling [3]. Once IHC steps are complete the resulting tissue is placed in a refractive index matching solution (RIMS), rendering tissue optically transparent and suitable for 3D imaging. All peripheral nerve segmentation and quantification was performed by the free software Image J Fiji [4].

Optimization in tissue clearing, immunohistochemistry for TC, image acquisition, and 3D segmentation have allowed for full 3D reconstruction and quantification of IENFs from the epidermis of rats. This technique provides the ability to measure 3D IENF swelling volumetrically, surface area, total length, individual branch lengths, number of branches, and mean-grey-intensity or integrated density of multiple co-localized markers from immunoreactive (IR) fluorescence. This developing technique allows for IENF quantification in $3 \mathrm{D}$ and shows the potential to become a powerful tool for researchers and clinicians. 
The limitation of measuring three-dimensional objects from two-dimensional images has largely been accepted when quantifying the density of intra-epidermal nerve fibers. Through a novel approach that involves enzyme dermal-epidermal separation, specialized processing, tissue clearing, immunohistochemistry, confocal imaging, and software segmentation we have developed a technique that allows for IENF 3D reconstruction and quantification. Our developing IENF protocol unleashes 2D restraints and provides full $3 \mathrm{D}$ visualization with accurate and whole quantification of IENF complexes.

\section{References:}

[1] G Lauria et al, European Journal of Neurology 12 (2005), p. 747.

[2] C Walzer, M Benathan, E Frenk, Journal of Investigative Dermatology 92(1) (1992), p. 78.

[3] B Yang et al, Cell 154(4) (2014), p. 945.

[4] J Schindelin et al, Nature Methods 9(7) (2012), p. 676.

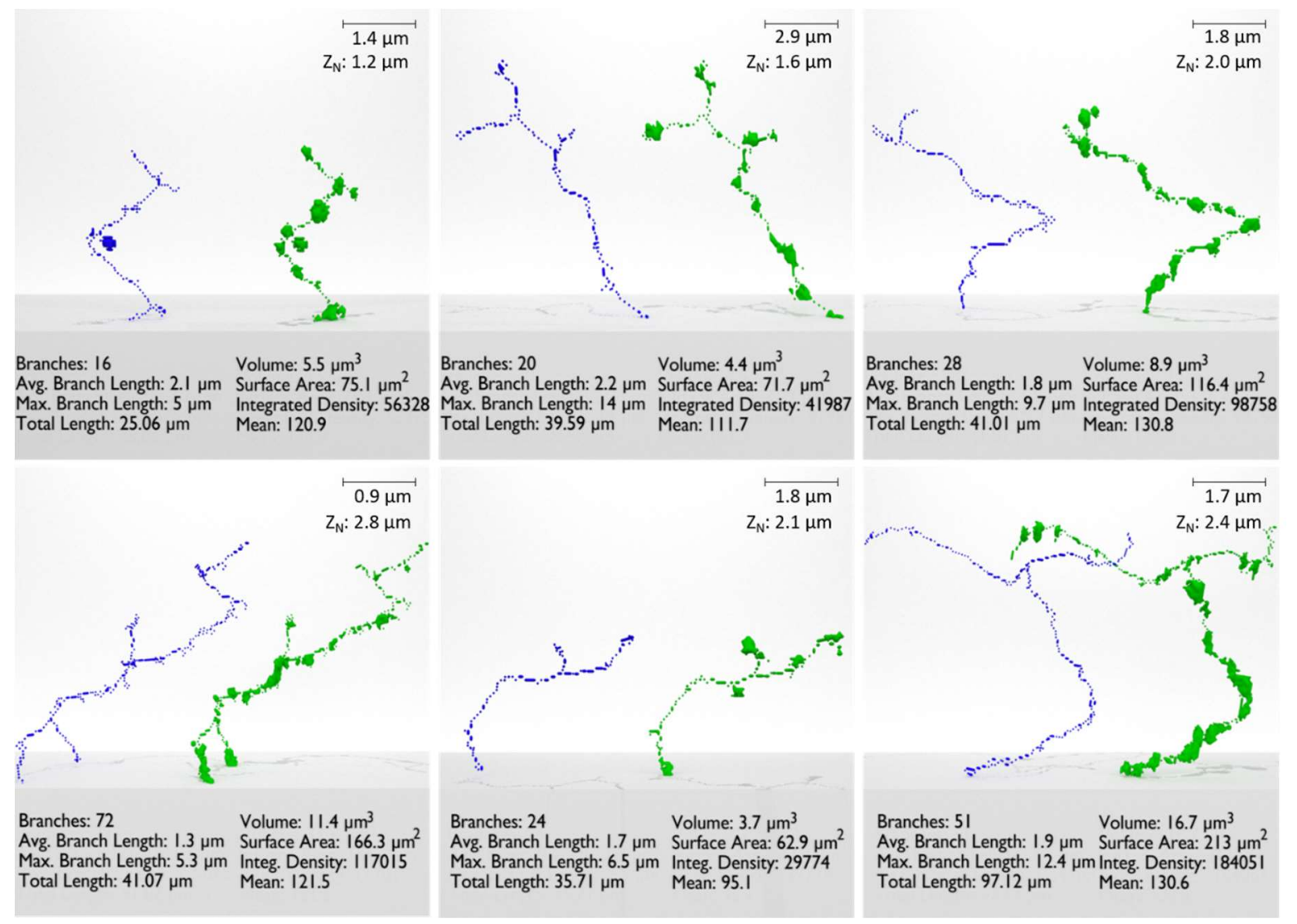

Figure 1. Six examples of individually isolated, 3D reconstructed, and quantified IENFs. Thick green objects are reconstructions based on selective nerve marker protein-gene-product 9.5 (PGP9.5) IR and used for mean-grey-intensity, integrated density, and volumetric measurements. Thin blue objects are skeletonized versions of PGP9.5 reconstructions and used for length and branching measurements. Scale bars calculated per image by dividing hand-traced 2D maximum branch length, in pixels, by software calculated 3D maximum branch length, in microns. The z-depth of traversing nerves $\left(Z_{N}\right)$ range from $1.2-2.8 \mu \mathrm{m}$. This provides a scale to approximate $3 \mathrm{D}$ to $2 \mathrm{D}$ standard conversion. 